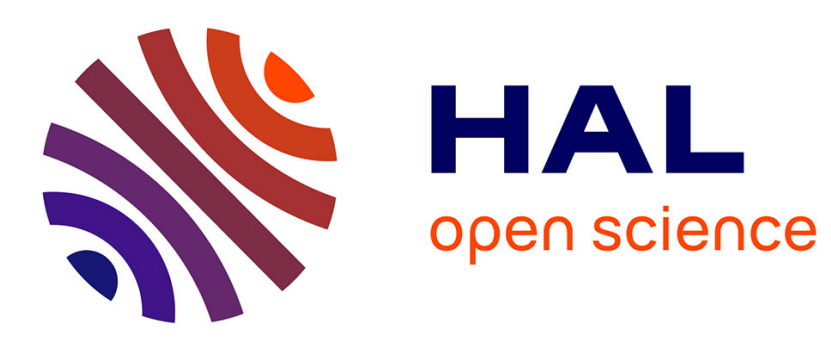

\title{
Crystal Structure Prediction could have helped the Experimentalists with Polymorphism in Benzamide!
}

Juergen Thun, Markus Schoeffel, Josef Breu

\section{To cite this version:}

Juergen Thun, Markus Schoeffel, Josef Breu. Crystal Structure Prediction could have helped the Experimentalists with Polymorphism in Benzamide!. Molecular Simulation, 2008, 34 (10-15), pp.13591370. 10.1080/08927020802208943 . hal-00515038

\section{HAL Id: hal-00515038 \\ https://hal.science/hal-00515038}

Submitted on 4 Sep 2010

HAL is a multi-disciplinary open access archive for the deposit and dissemination of scientific research documents, whether they are published or not. The documents may come from teaching and research institutions in France or abroad, or from public or private research centers.
L'archive ouverte pluridisciplinaire HAL, est destinée au dépôt et à la diffusion de documents scientifiques de niveau recherche, publiés ou non, émanant des établissements d'enseignement et de recherche français ou étrangers, des laboratoires publics ou privés. 


\section{Molecular Simulation}

Journal of

Experimental Nanoscience

- Taylor \& Francis

Taylor Sfrancis Group

\section{Crystal Structure Prediction could have helped the} Experimentalists with Polymorphism in Benzamide!

\begin{tabular}{|r|l|}
\hline Journal: & Molecular Simulation/Journal of Experimental Nanoscience \\
\hline Manuscript ID: & GMOS-2008-0025.R2 \\
\hline Journal: & Molecular Simulation \\
\hline Date Submitted by the & O2-May-2008 \\
\hline Complete List of Authors: & $\begin{array}{l}\text { Thun, Juergen; University of Bayreuth, Inorganic Chemsitry I } \\
\text { Schoeffel, Markus; University of Bayreuth, Inorganic Chemistry I } \\
\text { Breu, Josef; University of Bayreuth, Inorganic Chemistry I }\end{array}$ \\
\hline Keywords: & $\begin{array}{l}\text { Crystal Structure Prediction, Density Functional Theory, Benzamide, } \\
\text { Polymorphism, Atomic Point Charges }\end{array}$ \\
\hline &
\end{tabular}


000

\author{
Running heads (verso) A.N. Other and J. Bloggs or A.N. Other et al. \\ (recto) Journal title
}

\begin{abstract}
ARTICLE TYPE ('Full Paper')
Crystal Structure Prediction could have helped the Experimentalists with Polymorphism in Benzamide!
\end{abstract}

Juergen Thun, Markus Schoeffel and Josef Breu*

Inorganic Chemistry I, University of Bayreuth, Bayreuth, Germany

Benzamide was the first molecular material for which polymorphism was reported as long as 176 years ago. Unfortunately, due to very similar cell metrics leading to massive peak overlap, the metastable form reported by Liebig escaped structural characterization by XRD until recently. With the help of crystal structure prediction this old riddle of "Liebig's" polymorph of benzamide could have been solved many years earlier. Performing state of the art crystal structure prediction runs applying a commercial suite of programs (Cerius ${ }^{2}$ and the MS Modeling) identified both, the metastable and the thermodynamically stable phase reported by Liebig, as low energy packings.

Keywords: Crystal Structure Prediction; Density Functional Theory; Point Charges; Benzamide, Polymorphism

Title Page Footnote:

*Corresponding author. Email: josef.breu@uni-bayreuth.de 


\section{Introduction}

Polymorphism is a wide spread phenomenon occurring with many known crystalline materials. Polymorphism describes the ability of crystalline material to crystallise in more than one crystal structure [1]. The physical and chemical properties like melting point, solubility, and bioavailability may differ. The latter feature is of course of great interest in pharmaceutical research. As a consequence of these differing properties the crystal structure is relevant for patent licensing as well. The most famous and often cited example in this respect is the case of Ritonavir [2,3]. In most cases, an active pharmaceutical ingredient (API) is protected by a patent covering both, molecular and crystal structure(s). If additional crystal structures/metastable phases of this API can be obtained, this patent protection can be circumvented. Even a separate patent covering this new crystal structure can be applied for. In the dye industry the situation is comparable [4,5]. For these reasons a good knowledge about possible polymorphs is essential. Crystal structure prediction (CSP) represents a fast and cheap method to acquire a general idea of local energy minima on the lattice energy hyper surface. Several different techniques can be found in the literature and the success of these methods is checked regularly [6,7,8]. Rigid body calculations allow a very accurate description of the intermolecular potentials using distributed multipole analysis $[9,10,11]$. But these techniques are more costly and at the same time a view on the results presented here would suggest that the precision of the force field can not be improved significantly. Things become even more complicated when flexible bodies are used during the minimisation procedure. The use of quantum mechanically derived monopole charges is much less demanding and yields comparable accuracies in respect to lattice parameters, as will be shown later on. The energy ranking will be more exact using distributed multipoles [12] but for a first overview of possible polymorphs and their packing motifs monopole charges are adequate. Nevertheless, CSP still is a challenging and tricky task to perform, especially for the API molecules the pharmaceutical industry is interested in. But even small molecules may still pose challenges. Benzamide appears to be a very good benchmark for CSP because it is a small molecule with only one torsional degree of freedom (see Figure 1). In total, by now three polymorphs of this small molecule have been identified experimentally [13]. The lattice energies of two phases reported by Liebig (phase I and phase III) lie at close quarters and the plate like crystals of phase I have been regarded as to be the global minimum structure. Phase II reported by Davey et al. [16] appears to be a highly metastable or even transient packing. The task was to reproduce these known polymorphs by CSP. A state of the art approach was taken using different force fields (point charge models) in combinations with the commercial Cerius $^{2}$ [14] and MS Modeling suite of programs [15] for global optimisation. Of course, all predictions had been performed without implementing any knowledge about the different packing motifs found experimentally for these three polymorphs.

\section{Brief summary of crystallographic details}

All three polymorphs (see Table 1) show a similar hydrogen bonding motif $[16,17,13]$. Two benzamide molecules form hydrogen-bonded dimers and are connected by further hydrogen bonds to form long ribbons along the short axis of the unit cell (see Figure 2). Interestingly, despite the similar hydrogen bonding motifs, the $\pi-\pi$ interactions are quite different. In phase I a so called shifted $\pi$ stack is realised, while phase II and phase III show a herringbone pattern like arrangement (see Figure 3 ). So you can find all low energy $\pi$ - $\pi$ interaction motifs realised within the different crystal structures of the same molecule. One major difference lies in the number of symmetry independent molecules. While phase I and phase III contain only one independent molecule in the asymmetric unit cell phase II contains two independent 
molecules. CSP runs with more than one independent molecule still represent a challenge for the established algorithms [6].

\section{Computational methods}

\subsection{Method of generating the molecular structure and charge.}

\section{1. 1. Geometry.}

During the global optimisation applying a Monte Carlo (MC) algorithm as implemented in the Cerius $^{2}$ [14] and MS Modeling suite of programs [15], all molecules are held rigid. Therefore, in CSP runs for molecules that possess internal degrees of freedom, several different starting conformers should be used to get a reliable sampling over the whole potential energy surface. Since benzamide has only one torsional degree of freedom, low energy starting conformers could easily be identified by a grid search technique. The torsion angle $\varphi$ (see Figure 1) was modified from $0^{\circ}$ up to $90^{\circ}$ and a geometry optimisation of the gas phase molecules was performed with the Forcite module within the Material Studio software [15] using Gasteiger charges [18] and the Dreiding 2.21 force field [19]. Unsurprisingly, only one minimum structure could be found at $0^{\circ}$ torsion. As a second starting conformer the saddle point on the gas phase energy hyper surface was chosen which has a $90^{\circ}$ torsion. Please note, that these molecular structures used in the MC runs differ significantly in respect to the torsion angles from the experimentally found ones (about $30^{\circ}$ ). No bias regarding the molecular structure found experimentally was allowed in the CSP runs.

\section{1. 2. Choice of Force Field.}

Different density functional theory (DFT) methods had been used to derive several sets of partial charges that best represent the molecular electrostatic potential of benzamide. In Material Studio $\mathrm{DMol}^{3}[20,21]$ was used with a DNP basis set and the GGA-PBE functional and also the GGA-PW91 functional. The atomic charges were then fitted so as to reproduce the electrostatic potential (ESP) [22,23]. Additionally, ESP derived partial charges have been obtained in the same manner by using Gaussian03 [24] with a 6-311++G(d,p) basis set and the PBE1PBE[25] or the B3LYP hybrid functional [26,27], respectively. Both Gaussian03 calculations produced the same set of partial charges. Next, a quality check of the calculated sets of point charges in combination with different force fields had been performed using the crystal structure of the accurately known phase I as a benchmark. The molecules had been treated as flexible bodies during the geometry optimisation within the Forcite module and no further constraints had been introduced. The best results as judged by the deviation in cell constants could be obtained by using the Dreiding 2.21 force field in combination with the Gaussian03 point charges. Using the COMPASS force field or partial charges based on $\mathrm{DMol}^{3}$ calculations lead to larger deviations. The details can be seen in Table 2. When comparing the experimental lattice parameters with the parameters obtained after lattice energy minimizations deviations of less than 5\% were regarded acceptable. The good agreement achieved using the Gaussian03 set of partial charges in combination with the Dreiding 2.21 force is reassuring and thus this force field was used for all subsequent calculations. Special caution has been taken to assure that the cost function in both program suites applied in global optimisation was indeed identical. For instance, as Ewald summation was used throughout all geometry optimisations and lattice energy calculations for both Coulomb and dispersion forces, it was assured that the same set of accuracies $(0.000001$ $\mathrm{kcal} / \mathrm{mol}$ ) was applied. 


\section{2. Generating trial crystal structures.}

\section{2. 1. Cerius $^{2}$.}

A Monte Carlo simulated annealing algorithm [28,29,30,31] as implemented in the Cerius ${ }^{2}$ software suite was used. The nine most commonly observed space groups $\left(P 2_{1} / c, P \overline{1}\right.$, $P 22_{1} 22_{1}, P 2_{1}, C 2 / c, P b c a, P n m a, P n a 2_{1}$ and $\left.P b c n\right)$ were searched with one molecule in the asymmetric unit. The space groups $P \overline{1}, P 2_{1}, P 2_{1} / c, P 2_{1} 2_{1} 2_{1}$ and $P n a 2_{1}$ were also searched with $Z^{\prime}=2$. The space group $P b a 2$, the space group experimentally observed for phase II, is not available for CSP in the used Cerius ${ }^{2}$ suite of programs. Therefore, additionally a search in $P n a 2_{1}$ was performed. $P n a 2_{1}$ is a subgroup of $P b a 2$ and can be represented by a unit cell doubled along the c-axis. Consequently, this further search in $P n a 2_{1}$ had to be performed with $Z^{\prime}=4$. Rigid bodies were used throughout the simulated annealing procedure. Two different conformations with a $0^{\circ}$ or $90^{\circ}$ torsion angle $\varphi$, respectively, were used as rigid bodies. Because the balance between intra- and intermolecular energies was neglected during the MC runs, initially rather loosely packed trial crystal structures are generated. At least three independent repeats had been performed with each of the two different starting conformers until a convergence in low energy structures could be found. Each run consists of $7000 \mathrm{MC}$ steps and yielded a acceptance ratio of about $60 \%$.

\section{2. 2. MS Modeling.}

All above mentioned space groups had been searched as well with the Monte Carlo algorithm as implemented in the MS Modeling suite of programs. Additionally, the space group Pba2 had been examined by using $Z^{\prime}=2$. In all space groups at least three independent repeats had been performed with each of the two different starting conformers until a convergence in low energy structures was assured. As in the Cerius ${ }^{2}$ calculations, $7000 \mathrm{MC}$ steps had been performed and also yielded about $60 \%$ of acceptance.

\section{2. 3. Geometry optimisation.}

For both, the Cerius ${ }^{2}$ and the MS Modeling MC algorithms, a clustering algorithm limits the number of accepted crystal structures to 500 after each simulated annealing run. The geometry optimisation of 500 structures can be handled with ordinary cpu-power within a few hours or days depending on the degrees of freedom in the explored space group. The clustering of the structures is based on the similarity of their radial distribution functions. The molecular flexibility was introduced directly after the clustering and all accepted crystal structures were energy minimised with the force field described before. At this stage, all structural parameters like lattice parameters, molecular positions, orientations and conformations were relaxed. After geometry optimisation, the density of all crystal structures reaches $1.1-1.4 \mathrm{~g} \mathrm{~cm}^{-1}$ compared to $0.3-0.8 \mathrm{~g} \mathrm{~cm}^{-1}$ before the minimisation.

\section{2. 4. Ranking of structures.}

After a final clustering, the calculated crystal structures were ranked on the one hand according to their total energy and on the other hand according to their density. At least 20 structures with low energy and 20 structures with high density were also compared "by eye" attempting to identify the packing motifs of the experimentally known polymorphs. The unit cells of interesting predicted crystal structures were transformed into the standard settings applying LePage as implemented in Platon [32]. 


\section{Results and discussion}

\section{1. Prediction for $Z^{\prime}=1$}

\section{1. 1. Cerius $^{2}$ results.}

The predicted structures with the lowest lattice energies were found in the space group $P 2{ }_{1} / c$ and two structures clearly break away in the energy density plot (see Figure 4) with very similar energy and density. These two predicted structures are the most densely packed crystal structures and have the lowest achieved energy that have been found in all runs and they can be assigned to phase I and phase III, respectively (see Table 3). The details of the predicted and the experimentally observed packing motifs, a shifted $\pi$ stack for phase I and a herringbone pattern for phase III, are in close agreement (see Figure 5). The maximum error of the predicted lattice parameters amounts to $5.7 \%$ and also the experimentally observed torsion angles $\varphi$ were predicted very well within errors of about 8 and $5 \%$ for phase I and III, respectively. Please note, that the starting conformers used during $\mathrm{MC}$ exhibited either a $0^{\circ}$ or $90^{\circ}$ torsion angle $\varphi$ and the point charges had been calculated and fitted to the electrostatic potential of the gas phase minimum. Taking this into account, the agreement of predicted and experimental torsion angles is astonishing. It is expected, that a more accurate description of the electrostatic interactions could be achieved using distributed multipole moments. However, results recently published by Karamertzanis et al. show only slightly smaller deviations [33]. Even with our very simple charge model, the density and even torsions could be reproduced within nearly the same error limits. For a small molecule like benzamide, there seems to be no need for a more accurate description of the electrostatics, which of course would be much more time consuming. Even the energy ranking of both predicted structures is in agreement with experiment (see Table 3). The lowest energies of predicted structures in the other searched space groups had been around $-62 \mathrm{kcal} \mathrm{mol}^{-1}$ per molecule and the density was less than $1.28 \mathrm{~g} \mathrm{~cm}^{-3}$.

\section{1. 2. MS Modeling results.}

The experimentally observed polymorphs, phase I and phase III, could be identified as low energy/high density structures with the MS Modeling suite of programmes as well (see Figure 7). The predicted crystal structures compare very well with the experimentally observed ones (see Table 4 and Figure 8) and structural differences between the Cerius ${ }^{2}$ predicted structures and the MS Modeling predicted ones are very small (compare Table 3 and 4).

Also, a final single point energy calculation applying Cerius ${ }^{2}$ on phases I and III as predicted in MS Modeling yielded within calculational errors the same lattice energies as the Cerius ${ }^{2}$ predicted ones (compare Table 3 and Table 5) and the gradients were negligible. However, the absolute values between Cerius ${ }^{2}$ and MS Modeling differ by roughly one $\mathrm{kcal} / \mathrm{mol}$ as can be seen in Table 5. The difference occurs within the intramolecular part of the total energy. Although we intended to apply the very same force field parameters and precision in calculations, surprisingly the intramolecular fraction of the total energy is more positive within MS Modeling. Unfortunately, in the Version 4.0 of MS Modeling there is no force field editor for the Dreiding force field, which rendered a final analysis, of the origin of this divergence, impossible. This difference in lattice energies comes down to a different balance/weighting of inter- and intramolecular energies in Cerius ${ }^{2}$ and MS Modeling. This in turn might explain, why the global optimization runs identified different local minima (see below).

There is also a quite pronounced difference in the energy ranking of the generated crystal structures. While in the Cerius ${ }^{2}$ prediction phase I and III could be found on position one and two, respectively, the energy ranking position after the MS Modeling prediction has been 3 and 12, respectively. The insert of Figure 7 shows that many structures with a similar energy had been predicted, but the arrangement of the molecules is quite different. A density ranking 
placed both predicted phases I and III on position 30 for phase I and 13 for phase III, respectively. This result is not influenced by the molecular structure used in the prediction. The starting conformer with a $90^{\circ}$ torsion angle $\varphi$ yielded similar results. Taking a closer look on the predicted lowest energy structure pronounced differences in the packing motif as compared with the known phases are obvious, especially in respect to the hydrogen bonding pattern (see Figure 9a). This structure contains no dimers, the benzamide molecules only form hydrogen bonded chains along the short axis of the unit cell. This catamer synthon is frequently found for carbonic acids [34]. The contribution of the hydrogen bonding to the electrostatic energy is less attractive than in the above mentioned crystal structures of phase I and III, but the van der Waals interaction is slightly more favourable leading to a lower total energy. This suggests that the odds to crystallise this polymorph should increase in strongly hydrogen bonding solvents that are capable to break up the benzamide dimers in solution.

Interestingly, the most densely packed structure also contains chains of hydrogen bonded molecules along the short axis of the unit cell, but in this case some kind of a zig-zag orientation was realised (see Figure 9b). The hydrogen bonding part of the non-bonding energy again is less attractive as compared to the energies of the experimentally known phases I and III, because the strong hydrogen bonds within the benzamide dimers are not realised. Looking on the $\pi$ - $\pi$ interactions, a shifted $\pi$ stack had been realised for the lowest energy structure, while the highest density structure shows a tilted herringbone pattern arrangement. One $\mathrm{C}-\mathrm{H}$ bond is pointing to the middle of a neighbouring benzene ring. Nevertheless, the total energy of this structure is about $5 \mathrm{kcal} \mathrm{mol}^{-1}$ per molecule higher compared to the lowest energy structure.

\section{1. 3. Discussion}

Although the methods should be nearly identical, the results of the calculations with the two programs appear quite diverse and might lead to different conclusions on either the choice of method or more generally on the usefulness of crystal structure prediction. On a first glance, Cerius $^{2}$ appears to give a much clearer picture: The known phases I and III are found as the lowest energy crystal structures - a result that would be very useful if it was general and reproducible also for other molecules. The same set of calculations performed within the MS Modeling suite of programs also finds phase I and III, but quite far up the list of structures - a less useful result? Apparently, the global optimisation of MS Modeling is more thorough and is capable of identifying additional low energy/high density packings. As mentioned above, a possible reason for the better performance of the global search might be the differing balance/weighting of intra- and intermolecular contributions to the total energy.

A final single point energy calculation of the MS Modeling predicted lowest energy structure within the Cerius ${ }^{2}$ suite shows clearly, that the global optimisation within Cerius ${ }^{2}$ seems to be incomplete. This structure is assigned the lowest energy also within Cerius ${ }^{2}$ (-66.053 kcal $\mathrm{mol}^{-1}$ per molecule) and hence Cerius ${ }^{2}$ failed to identify this valid global minimum.

In summary, two alternative conclusions may be drawn:

On one hand, the experimental screening may not be exhausted. This would be the more expedient result for experimentalists and might possibly lead to the identification of another polymorph. This result would pass the ball back to the experimentalists and ask for further polymorph screening experiments.

On the other hand, if the experimental screening has indeed been exhausted, it would suggest that the cost function applied is not sufficiently precise and therefore the ranking of phase I and III is wrong. This would pass the ball into the field of the prediction community and asks for further improvements of force fields.

Nevertheless, could this excellent agreement of the predicted and the experimentally observed crystal structures have helped to earlier identify the metastable polymorph reported by Liebig? This phase III managed to "hide" behind the stable phase I for such a long time 
because the metric of the unit cell is very similar to the one of phase I and peak overlap is severe [13]. Since phase III can only be obtained as a 50:50 mixture with phase I, it was therefore simply overlooked.

Obviously, even such small deviations in cell axes and relative coordinates as observed in this CSP greatly affects both, the positions and the intensities of the diffraction peaks. The differences between the calculated and experimentally observed X-ray powder diffraction pattern (PXRD) are immense (Figure 6). It is hard to imagine that any person or algorithm would have been able to identify phase III in the overlapping experimental PXRD trace of the mixture accessible in the experiment with the help of the PXRD calculated using the predicted structure. The lattice energy as calculated using classical force fields is inappropriate to be used as the only cost function for structure solution in real space. However, it is obvious from the calculated diffraction patterns that the very first low angle peaks (around $5^{\circ} 2 \theta$ ) of the two predicted phases are overlapping while in the experimental trace a shoulder is visible. Consequently, with some trust in the CSP results it could have appeared that the experimental trace might not be pure phase I as has been assumed for such a long time but a mixture of the two low energy structures.

\section{2. Prediction for $Z^{\prime}>1$}

\section{2. 1. Cerius $^{2}$ results.}

The experimentally observed crystal structure of phase II could not been found in either of the prediction runs in Pna $2_{1}$ with $Z^{\prime}=4$ using starting conformers with $0^{\circ}$ or $90^{\circ}$ torsion angle. Therefore, starting conformers with torsions as observed in the known packing motif of phase II had been included as a bias in the MC runs, again without success. Additionally, benzamide dimers and pairs of dimers as observed in the crystal structure of phase II had also been applied as rigid units during the Monte Carlo algorithm. The ESP-derived partial charges had been calculated for these oligomeric units with Gaussian03 and the above mentioned basis set and functionals. Despite all these additional efforts that have been severely biased by knowledge from the experimentally determined structure, phase II could not been obtained by CSP.

A search in the space group $P \overline{1}$ with $Z^{\prime}=2$ found phase I and phase III. The predicted crystal structures fit quite well with experimental structures, but the prediction of phase III showed bigger deviations as compared to the runs in space group $P 2_{1} / c$ and the energies were higher (see Table 6). These calculations showed, that a crystal structure prediction with more than one molecule within the asymmetric unit is in principle possible for benzamide.

\section{2. 2. MS Modeling results.}

Although in MS Modeling, prediction runs in space group Pba2 and Z' $=2$ were accessible, phase II could not be identified either. The same biased rigid units as described in the preceding paragraph had been used, again without any mentionable results.

However, as with Cerius ${ }^{2}$, phases I and III can also be identified in a search in the space group $P \overline{1}$ and two independent molecules within the asymmetric unit (see Table 7). This suggests that the problems encountered in predicting phase II are not necessarily related with the fact that one has to deal with $Z^{\prime}=2$. Preliminary results on quenched MD simulation runs starting with the experimentally known structure did not yield additional insights.

\section{2. 3. A closer look at phase II.}

To shed some light into the difficulties encountered with the prediction of phase II a simple static energy optimisation of the known phase II had been performed. The crystal structure of phase II had to be determined with a diffraction trace that suffered from two major draw backs: A large and broad background caused by the solvent and peak overlap with the 
majority phase I (82.7\% [17]). Therefore, it may be anticipated that the details of the crystal structure are less reliable. And indeed, the experimental crystal structure represents a saddle point in respect to the lattice energy as defined by the force field. Especially the van der Waals energy is highly positive (+66.75 kcal mol${ }^{-1}$ per molecule). Apparently there are some non-bonding contacts that are too short leading to highly repulsive forces that direct the static minimisation into a wrong secondary minimum. To avoid this, a stepwise approach was used to relax the experimentally known crystal structure of phase II. In the first step, all C-H bonds had been shortened to $0.9 \AA$ and additionally the lattice parameters of the $a$ and $b$ axis had been inflated by about $10 \%$. In the following two rigid body optimisations, no internal energies had been considered. During the first geometry optimisation only the orientation of the rigid bodies had been allowed to relax within a fixed unit cell and during the second geometry optimisation additionally the lattice parameters had been optimised. A third optimisation run relaxed the molecules within the fixed unit cell and finally all parameters had been optimised. The final energy minimised crystal structure is close to the experimental structure. However, the deviation in the $b$ axis still amounts to $10.5 \%$. This suggests that the dimers can not be accurately represented by standard force fields and this may contribute to the difficulties encountered in CSP of phase II. Apparently, phase II is very sensitive to small changes in the molecular geometry and therefore CSP is more severely hampered by inaccuracies in the force field and the negligence of balance of the inter-/intramolecular interactions during the MC runs. The calculated energy of phase II is very similar to the energies of phase I and phase III (see Table 8) and also the energy ranking is in good agreement with experimental results. Please note, that the energies published recently had been calculated without taking the internal energies into account (compare Thun et al. [13]). An overlay of the optimised and the experimental crystal structure shows the good agreement after the minimisation. In the relaxed structure, the hydrogen bonding within a benzamide dimer is more distinct as compared to the experimental structures due to a more favourable orientation of the two amide groups (see Figure 10 and compare the change in the torsion angles $\varphi_{1}$ and $\varphi_{2}$ of both independent molecules in Table 8). In Figure 11 the herringbone motif is shown before and after refinement. After minimisation, the $\pi$-systems of two dimers lie perfectly above each other and a $\mathrm{C}-\mathrm{H}$ group of one benzene ring points to the centre of a neighbouring benzene system. Nevertheless, also this relaxed structure could not been found in either of the performed simulations. Since the agreement in lattice constants of experimental and optimized phase II is mediocre, expectedly a comparison of the simulated and experimental powder pattern also shows pronounced differences (see figure 12).

But clearly, phase II is a valid local minimum in the energy landscape of benzamide packings. In respect to energy it is close enough to phase I and phase III in order to be identified in the CSP runs. At the moment it is hard to say whether the problems with CSP of this known polymorph are related to the uncertainties in the force field or the difficulties encountered in global optimisation with $Z^{\prime}=2$.

\section{Conclusion}

This paper presents the successful prediction of two benzamide polymorphs. Using crystal structure prediction, the metastable polymorph described by Wöhler and Liebig 176 years ago could have been solved many years earlier. Within one day of calculation the structure of this metastable phase III would have been predicted within the Cerius ${ }^{2}$ suite of programs. Applying the Material Studio Modeling suite of programs, phase I and phase III could be identified as well. However, many more packings with comparable energy and/or density are predicted rendering the identification of the experimentally known structures tricky. The lowest energy structure found with MS Modeling is not yet known for benzamide but represents a well known structure motif for carbonic acids. Additional experimental 
Reference List

[1] Bernstein, J., Polymorphism in Molecular Crystals, Oxford University Press, Oxford, 2002.

[2] S.R. Chemburkar, J. Bauer, K. Deming, H. Spiwek, K. Patel, J. Morris, R. Henry, S. Spanton, W. Dziki, W. Porter, J. Quick, P. Bauer, J. Donaubauer, B.A. Narayanan, M. Soldani, D. Riley, and K. McFarland, Dealing with the impact of ritonavir polymorphs on the late stages of bulk drug process development, Org.Process Res.Dev. 4 (2000), pp. 413-417.

[3] H. Nowell and S.L. Price, Validation of a search technique for crystal structure prediction of flexible molecules by application to piracetam, Acta Cryst.B 61 (2005), pp. 558-568.

[4] M.U. Schmidt, C. Buchsbaum, J.M. Schnorr, D.W.M. Hofmann, and M. Ermrich, Pigment-Orange-5: crystal structure determination from a non-indexed $X$-ray powder diagram, Z.Kristallogr. 222 (2007), pp. 30-33.

[5] M.U. Schmidt, R.E. Dinnebier, and H. Kalkhof, Crystal engineering on industrial diaryl pigments using lattice energy minimizations and X-ray powder diffraction, J.Phys.Chem.B 111 (2007), pp. 9722-9732.

[6] G.M. Day, W.D.S. Motherwell, H.L. Ammon, S.X.M. Boerrigter, R.G. Della Valle, E. Venuti, A. Dzyabchenko, J.D. Dunitz, B. Schweizer, B.P. van Eijck, P. Erk, J.C. Facelli, V.E. Bazterra, M.B. Ferraro, D.W.M. Hofmann, F.J.J. Leusen, C. Liang, C.C. Pantelides, P.G. Karamertzanis, S.L. Price, T.C. Lewis, H. Nowell, A. Torrisi, H.A. Scheraga, Y.A. Arnautova, M.U. Schmidt, and P. Verwer, A third blind test of crystal structure prediction, Acta Cryst.B 61 (2005), pp. 511-527. 
[7] J.P.M. Lommerse, W.D.S. Motherwell, H.L. Ammon, J.D. Dunitz, A. Gavezzotti, D.W.M. Hofmann, F.J.J. Leusen, W.T.M. Mooij, S.L. Price, B. Schweizer, M.U. Schmidt, B.P. van Eijck, P. Verwer, and D.E. Williams, A test of crystal structure prediction of small organic molecules, Acta Cryst.B 56 (2000), pp. 697-714.

[8] W.D.S. Motherwell, H.L. Ammon, J.D. Dunitz, A. Dzyabchenko, P. Erk, A. Gavezzotti, D.W.M. Hofmann, F.J.J. Leusen, J.P.M. Lommerse, W.T.M. Mooij, S.L. Price, H. Scheraga, B. Schweizer, M.U. Schmidt, B.P. van Eijck, P. Verwer, and D.E. Williams, Crystal structure prediction of small organic molecules: a second blind test, Acta Cryst.B 58 (2002), pp. 647-661.

[9] U. Koch and A.J. Stone, Conformational dependence of the molecular charge distribution and its influence on intermolecular interactions, J.Chem.Soc.Faraday Trans. 92 (1996), pp. 1701-1708.

[10] A.J. Stone and M. Alderton, Distributed multipole analysis - Methods and applications (Reprinted from Molecular Physics, vol 56, pg 1047-1064, 1985), Mol.Phys. 100 (2002), pp. 221-233.

[11] A.J. Stone, Distributed Multipole Analysis, Or How to Describe A Molecular ChargeDistribution, Chem.Phys.Lett. 83 (1981), pp. 233-239.

[12] G.M. Day, W.D.S. Motherwell, and W. Jones, A strategy for predicting the crystal structures of flexible molecules: the polymorphism of phenobarbital, Phys.Chem.Chem.Phys. 9 (2007), pp. 1693-1704.

[13] J. Thun, L. Seyfarth, J. Senker, R.E. Dinnebier, and J. Breu, Polymorphism in benzamide: Solving a 175-year-old riddle, Angew.Chem.Int.Ed. 46 (2007), pp. 67296731.

[14] Accerlys, Cerius², Release 4.9, Accelrys Software, Inc., San Diego (2003)

[15] Accerlys, Material Studio, Release 4.0, Accelrys Software, Inc., San Diego (2006)

[16] N. Blagden, R. Davey, G. Dent, M. Song, W.I.F. David, C.R. Pulham, and K. Shankland, Woehler and Liebig revisited: A small molecule reveals its secrets - The crystal structure of the unstable polymorph of benzamide solved after 173 years, Cryst.Growth Des. 5 (2005), pp. 2218-2224.

[17] W.I.F. David, K. Shankland, C.R. Pulham, N. Blagden, R.J. Davey, and M. Song, Polymorphism in benzamide, Angew.Chem.Int.Ed. 44 (2005), pp. 7032-7035.

[18] J. Gasteiger and M. Marsili, Iterative Partial Equalization of Orbital Electronegativity A Rapid Access to Atomic Charges, Tetrahedron 36 (1980), pp. 3219-3228.

[19] S.L. Mayo, B.D. Olafson, and W.A. Goddard, Dreiding - A Generic Force-Field for Molecular Simulations, J.Phys.Chem. 94 (1990), pp. 8897-8909.

[20] B. Delley, An All-Electron Numerical-Method for Solving the Local Density Functional for Polyatomic-Molecules, J.Chem.Phys. 92 (1990), pp. 508-517. 
[25] J.P. Perdew, K. Burke, and M. Ernzerhof, Generalized gradient approximation made simple, Phys.Rev.Lett. 77 (1996), pp. 3865-3868.

[26] A.D. Becke, Density-Functional Thermochemistry .3. the Role of Exact Exchange, J.Chem.Phys. 98 (1993), pp. 5648-5652.

[27] A.D. Becke, A New Mixing of Hartree-Fock and Local Density-Functional Theories, J.Chem.Phys. 98 (1993), pp. 1372-1377.

[28] H.R. Karfunkel and R.J. Gdanitz, Abinitio Prediction of Possible Crystal-Structures for General Organic-Molecules, J.Comput.Chem. 13 (1992), pp. 1171-1183.

[29] H.R. Karfunkel, F.J.J. Leusen, and R.J. Gdanitz, The ab initio prediction of yet unknown molecular crystal structures by solving the crystal packing problem, J.Computer-Aided Mater.Design 1 (1994), pp. 177-185.

[30] R.J. Gdanitz, Prediction of Molecular-Crystal Structures by Monte-Carlo Simulated Annealing Without Reference to Diffraction Data, Chem.Phys.Lett. 190 (1992), pp. 391396.

[31] P. Verwer and F.J.J. Leusen, Computer simulation to predict possible crystal polymorphs, Rev.Comput.Chem. 1998), pp. 327-365.

[32] A.L. Spek, Single-crystal structure validation with the program PLATON, J.Appl.Cryst. 36 (2003), pp. 7-13. 
[33] P.G. Karamertzanis and S.L. Price, Energy minimization of crystal structures containing flexible molecules, Journal of Chemical Theory and Computation 2 (2006), pp. 11841199.

[34] Desiraju, G. R., The Crystal as a Supramolecular Entity, John Wiley \& Sons Ltd., Chichester, 1996. 


\section{Page 13 of 33}

Table 1. Crystallographic details of phase I [17], phase II [17] and phase III [13].

\begin{tabular}{llll}
\hline & Phase I & Phase II & Phase III \\
\hline$a / \AA$ & 5.607 & $17.4317(2)$ & $5.0551(1)$ \\
$b / \AA$ & 5.046 & $14.1944(3)$ & $5.5141(1)$ \\
$c / \AA$ & 22.053 & $4.977(1)$ & $22.9565(9)$ \\
$\beta /{ }^{\circ}$ & 90.66 & - & $101.292(2)$ \\
${\text { Volume } / \AA^{3}}^{3}$ & 624.25 & $1231.4(3)$ & $627.50(3)$ \\
Space group & $P 2 / c$ & $P b a 2$ & $P 2_{1} / c$ \\
$Z$ & 1 & 2 & 1 \\
$Z$ & 4 & 8 & 4 \\
\hline
\end{tabular}


Table 2. Relative energies and unit cell parameters ${ }^{\mathrm{a}}$ of the observed and energy minimised crystal structures of phase I using Gaussian03 PBE1PBE ESP-charges (Gaus) and DMol ${ }^{3}$ PBE ESP-charges (DMol) in combination with the Dreiding 2.21 (drei) and COMPASS (com) force field within MS Modeling

\begin{tabular}{lrrrrrr}
\hline & $a / \AA$ & \multicolumn{1}{c}{$b / \AA$} & $c / \AA$ & \multicolumn{1}{l}{$\beta /^{\circ}$} & $\rho / \mathrm{g} \mathrm{cm}^{-3}$ & $\begin{array}{l}\text { Energy } \\
\mathrm{kcal} \mathrm{mol}^{-1}\end{array}$ \\
\hline Obs. & 5.607 & 5.046 & 22.053 & 90.66 & 1.287 & \\
Gaus $_{\text {drei }}$ & -2.2 & -2.3 & 4.4 & 0.7 & 1.293 & -63.859 \\
DMol $_{\text {drei }}$ & 6.6 & 1.8 & -2.1 & 1.7 & 1.285 & -48.388 \\
Gaus $_{\text {com }}$ & -8.6 & 14.7 & -1.2 & -11.3 & 1.261 & -80.207 \\
DMol $_{\text {com }}$ & -3.8 & 2.1 & 0.0 & -5.3 & 1.223 & -33.048 \\
\hline
\end{tabular}

${ }^{\mathrm{a}}$ Experimental lattice parameters and, in italics, lattice parameters for minimised structures given as a percentage difference from the experimental values.

${ }^{\mathrm{b}}$ Energy per molecule. 


\section{Page 15 of 33}

Table 3. Comparison of the predicted and observed structural parameters and total energies for phase I and phase III (Cerius ${ }^{2}$ calculation).

\begin{tabular}{lllllll}
\hline Parameter & $\begin{array}{l}\text { Phase I } \\
\text { Cerius }^{2}\end{array}$ & Exp. & $\Delta / \%$ & $\begin{array}{l}\text { Phase III } \\
\text { Cerius }^{2}\end{array}$ & Exp. & $\Delta / \%$ \\
\hline$a / \AA$ & 5.318 & 5.607 & -5.2 & 4.902 & 5.055 & -3.0 \\
$b / \AA$ & 4.905 & 5.046 & -2.8 & 5.232 & 5.514 & -5.1 \\
$c / \AA$ & 23.315 & 22.053 & 5.7 & 23.778 & 22.957 & 3.6 \\
$\beta /{ }^{\circ}$ & 91.71 & 90.66 & 1.2 & 95.75 & 101.29 & -5.5 \\
$\rho / \mathrm{g} \mathrm{cm}^{-3}$ & 1.323 & 1.287 & 2.8 & 1.326 & 1.282 & 3.4 \\
$\varphi /^{\circ}$ & 30.54 & 33.20 & -8.0 & 30.18 & 28.84 & 4.6 \\
Energy $^{a} /$ & -65.132 & & & -65.055 & & \\
kcal mol $^{-1}$ & & & & & &
\end{tabular}


Table 4. Comparison of the predicted and observed structural parameters and total energies for phase I and phase III (MS Modeling 4.0 calculation).

\begin{tabular}{|c|c|c|c|c|c|c|c|c|}
\hline \multirow[t]{2}{*}{ Parameter } & \multicolumn{3}{|l|}{ Phase I } & \multicolumn{3}{|l|}{ Phase III } & \multirow{2}{*}{$\begin{array}{l}\text { lowest } \\
\text { energy } \\
\text { structure }\end{array}$} & \multirow{2}{*}{$\begin{array}{l}\text { highest } \\
\text { density } \\
\text { structure }\end{array}$} \\
\hline & MS Mod. & Exp. & $\Delta / \%$ & MS Mod. & Exp. & $\Delta / \%$ & & \\
\hline$a / \AA$ & 5.322 & 5.607 & -5.1 & 4.904 & 5.055 & -3.0 & 3.477 & 11.787 \\
\hline$b / \AA$ & 4.905 & 5.046 & -2.8 & 5.232 & 5.514 & -5.1 & 7.795 & 5.914 \\
\hline$c / \AA$ & 23.316 & 22.053 & 5.7 & 23.776 & 22.957 & 3.6 & 18.912 & 12.777 \\
\hline$\beta /^{\circ}$ & 91.66 & 90.66 & 1.1 & 95.83 & 101.29 & -5.4 & 145.06 & 137.37 \\
\hline$\rho / \mathrm{g} \mathrm{cm}^{-3}$ & 1.301 & 1.287 & 1.1 & 1.326 & 1.282 & 3.4 & 1.302 & 1.334 \\
\hline$\varphi /^{\circ}$ & 30.72 & 33.20 & -7.5 & 30.17 & 28.84 & 4.6 & 13.49 & 21.27 \\
\hline $\begin{array}{l}\text { Energy }{ }^{a} \\
\text { kcal mol }^{-1}\end{array}$ & -64.065 & & & -64.052 & & & -65.070 & -59.858 \\
\hline
\end{tabular}

${ }^{\mathrm{a} E n e r g y ~ p e r ~ m o l e c u l e . ~}$ 


\section{Page 17 of 33}

Table 5. Single point intra- and intermolecular energies of phases I and III as predicted in MS Modeling.

\begin{tabular}{lllll}
\hline $\begin{array}{l}\text { Energy } \\
\text { kcal mol }^{\mathrm{a}}\end{array}$ & $\begin{array}{l}\text { Phase I } \\
\text { MS Mod }\end{array}$ & Cerius $^{2}$ & $\begin{array}{l}\text { Phase III } \\
\text { MS Mod. }\end{array}$ & Cerius $^{2}$ \\
\hline Intramoleculare & 8.478 & 7.411 & 8.451 & 7.448 \\
Bond & 1.974 & 1.776 & 1.982 & 1.753 \\
Angle & 2.948 & 2.547 & 2.945 & 2.565 \\
Torsion & 3.428 & 2.958 & 3.389 & 2.993 \\
inversion & 0.128 & 0.130 & 0.135 & 0.137 \\
Intermoleculare & -72.543 & -72.543 & -72.503 & -72.503 \\
Hydrogen bond & -6.744 & -6.744 & -6.735 & -6.735 \\
Van der Waals & 2.855 & 2.855 & 2.821 & 2.821 \\
Electrostatic & -68.654 & -68.654 & -68.589 & -68.589 \\
Total & -64.065 & -65.132 & -64.052 & -65.055 \\
\hline${ }^{\mathrm{a} E n e r g y ~ p e r ~ m o l e c u l e ~}$ & & &
\end{tabular}

${ }^{\mathrm{a}}$ Energy per molecule. 
Table 6. Comparison of the predicted and observed structural parameters and total energies for phase I and phase III in $P \overline{1}$ (Cerius ${ }^{2}$ calculation).

\begin{tabular}{lllllll}
\hline Parameter & $\begin{array}{l}\text { Phase I } \\
\text { Cerius }^{2}\end{array}$ & Exp. & $\Delta / \%$ & $\begin{array}{l}\text { Phase III } \\
\text { Cerius }^{2}\end{array}$ & Exp. & $\Delta / \%$ \\
\hline$a / \AA$ & 5.559 & 5.607 & -0.0 & 4.921 & 5.055 & -2.7 \\
$b / \AA$ & 4.923 & 5.046 & -2.4 & 5.207 & 5.514 & -5.6 \\
$c / \AA$ & 23.132 & 22.053 & 4.9 & 25.044 & 22.957 & 9.1 \\
$\beta /{ }^{\circ}$ & 91.64 & 90.66 & 1.0 & 93.98 & 101.29 & -7.7 \\
$\rho / \mathrm{g} \mathrm{cm}^{-3}$ & 1.272 & 1.287 & -1.2 & 1.257 & 1.282 & -2.0 \\
$\varphi /^{\circ}$ & 30.76 & 33.20 & -7.3 & 29.91 & 28.84 & 3.7 \\
Energy $^{a} /$ & -64.223 & & & -63.348 & & \\
kcal mol $^{-1}$ & & & & & & \\
${ }^{2}$ Energy per molecule. & & & &
\end{tabular}

19

20 


\section{Page 19 of 33}

Table 7. Comparison of the predicted and observed structural parameters and total energies for phase I and phase III in $P \overline{1}$ (MS Modeling calculation).

\begin{tabular}{lllllll}
\hline Parameter & Phase I & & \multicolumn{3}{c}{ Phase III } \\
& MS Mod & Exp. & $\Delta / \%$ & MS Mod & Exp. & $\Delta / \%$ \\
\hline$a / \AA$ & 5.329 & 5.607 & -5.0 & 4.922 & 5.055 & -2.6 \\
$b / \AA$ & 4.907 & 5.046 & -2.8 & 5.451 & 5.514 & -1.1 \\
$c / \AA$ & 23.256 & 22.053 & 5.5 & 23.730 & 22.957 & 3.4 \\
$/{ }^{\circ}$ & 91.74 & 90.66 & 1.2 & 91.72 & 101.29 & -9.4 \\
$\rho /$ cm $^{-3}$ & 1.324 & 1.287 & 2.9 & 1.313 & 1.282 & 2.4 \\
$\varphi /{ }^{\circ}$ & 33.15 & 33.20 & 0.0 & 32.98 & 28.84 & 14.4 \\
Energy $^{a} /$ & -64.052 & & & -63.335 & & \\
kcal mol $^{-1}$ & & & & & & \\
\hline
\end{tabular}

${ }^{a}$ Energy per molecule. 
Table 8. Cell parameters of the observed and energy minimised crystal structure of phase II using Gaussian03 PBE1PBE ESP-charges, the Dreiding 2.21 force field and a stepwise approach.

\begin{tabular}{lllc}
\hline Parameter & Cerius $^{2}$ & Exp. & $\Delta / \%$ \\
\hline$a / \AA$ & 17.095 & 17.432 & -1.9 \\
$b / \AA$ & 15.684 & 14.194 & 10.5 \\
$c / \AA$ & 5.151 & 4.977 & 3.5 \\
$\rho / g^{\circ} \mathrm{cm}^{-3}$ & 1.165 & 1.307 & -10.9 \\
$\varphi_{1} /^{\circ}$ & 24.68 & 38.77 & 36.3 \\
$\varphi_{2} /^{\circ}$ & 27.58 & 13.72 & 101.0 \\
Energy $^{a} /$ & -64.355 & $116.677^{b}$ & \\
kcal mol $^{-1}$ & & & \\
\hline
\end{tabular}

${ }^{\mathrm{a}}$ Energy per molecule.

${ }^{\mathrm{b}}$ Single point energy calculation in Cerius ${ }^{2}$ prior to lattice energy minimisation. 
Figure 3. A projection along the ribbon axes shows the $\pi-\pi$ interactions in the different forms. a) phase I, view along [010]; b) phase II, view along [001]; c) phase III, view along [100].

Figure 4. Energy-density plot in the space group $P 2{ }_{1} / c$ (Cerius ${ }^{2}$ runs). Packings corresponding to phase I and phase III are circled.

Figure 5. Overlay of the Cerius ${ }^{2}$ predicted (red) and observed (green) dimers of a) phase I and b) phase III.

Figure 6. Experimental powder diffraction pattern (blue) combined with the calculated diffraction data of the predicted phases I (red) and III (green).

Figure 7. Energy-density plot in the space group $P 2_{1} / c$. (MS Modeling runs). The inset shows a close up of the high density and low energy region. Packings corresponding to phase I and phase III are circled.

Figure 8. Overlay of the MS Modeling predicted (red) and observed (green) dimers of a) phase I and b) phase III.

Figure 9. Hydrogen bonding pattern in the predicted structures with a) the lowest energy and b) the highest density.

Figure 10. Overlay of the optimised (red) and experimental (green) unit cell of phase II.

Figure 11. View perpendicular onto a pair of dimers of benzamide as found in the crystal structure of phase II before (green) and after (red) energy minimisation.

Figure 12. Simulated powder pattern of the minimised structure and ticks (red) of the experimentally known phase II.

\author{
Special Issue: Molecular Simulation Materials Studio special issue
}




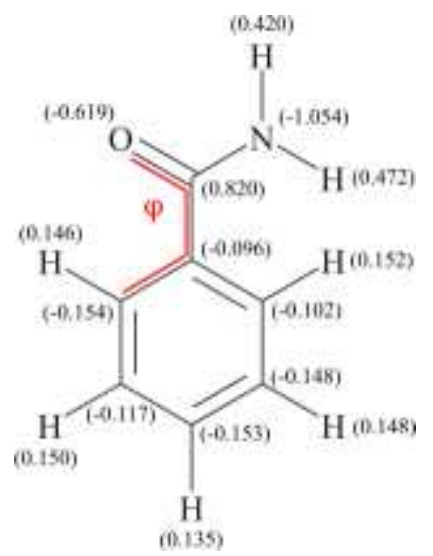

Figure 1. A benzamide molecule showing the torsion angle $\varphi$ and the Gaussian03 calculated partial charges in brackets.

$6 \times 8 \mathrm{~mm}(600 \times 600 \mathrm{DPI})$ 
a)

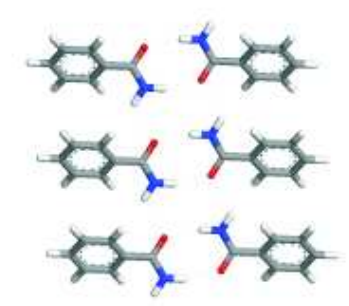

b)

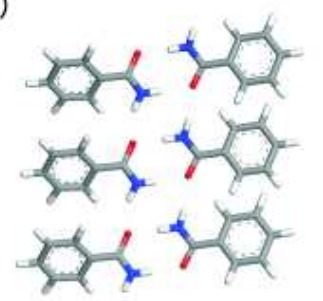

c)

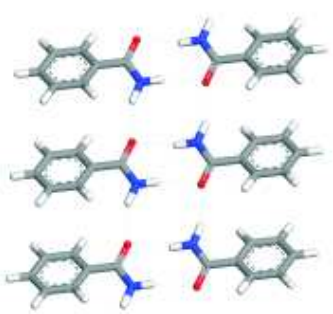

Figure 2. Hydrogen bonded networks. View along the short axis of the unit cells. a) phase I; b) phase II; c) phase III. Dashed blue lines show the hydrogen bonds.

$29 \times 7 \mathrm{~mm}(600 \times 600 \mathrm{DPI})$ 
a)

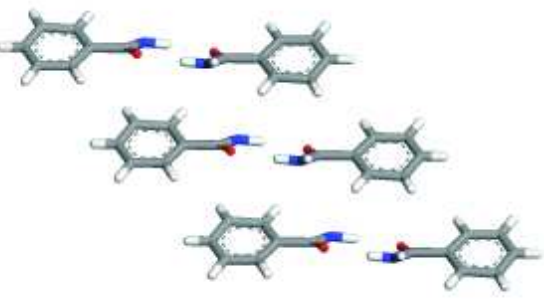

b)

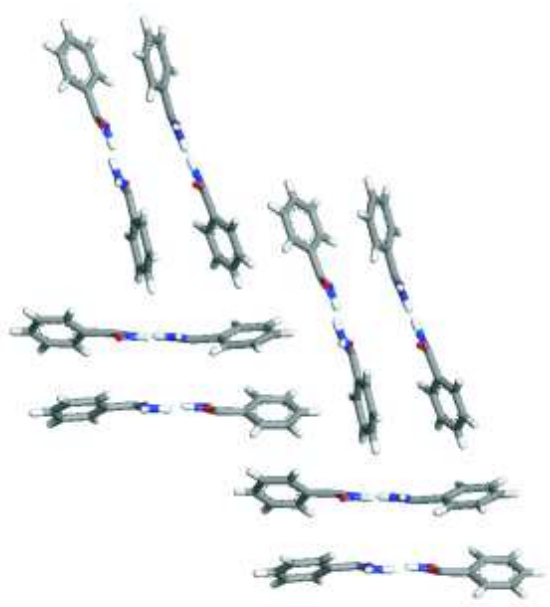

c)

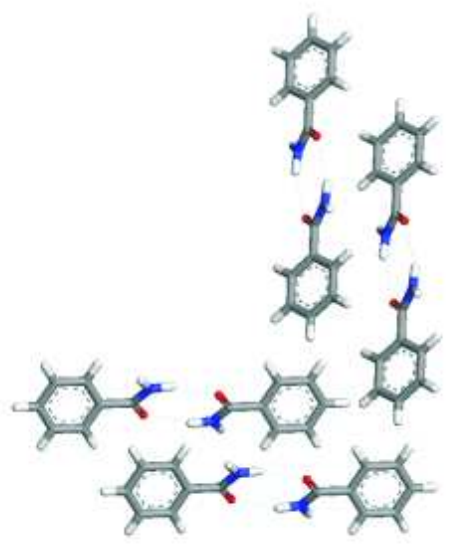

Figure 3. A projection along the ribbon axes shows the $\Pi \hat{A} \square \Pi$ interactions in the different forms. a) phase I, view along [010]; b) phase II, view along [001]; c) phase III, view along [100]. $18 \times 49 \mathrm{~mm}(400 \times 400 \mathrm{DPI})$ 


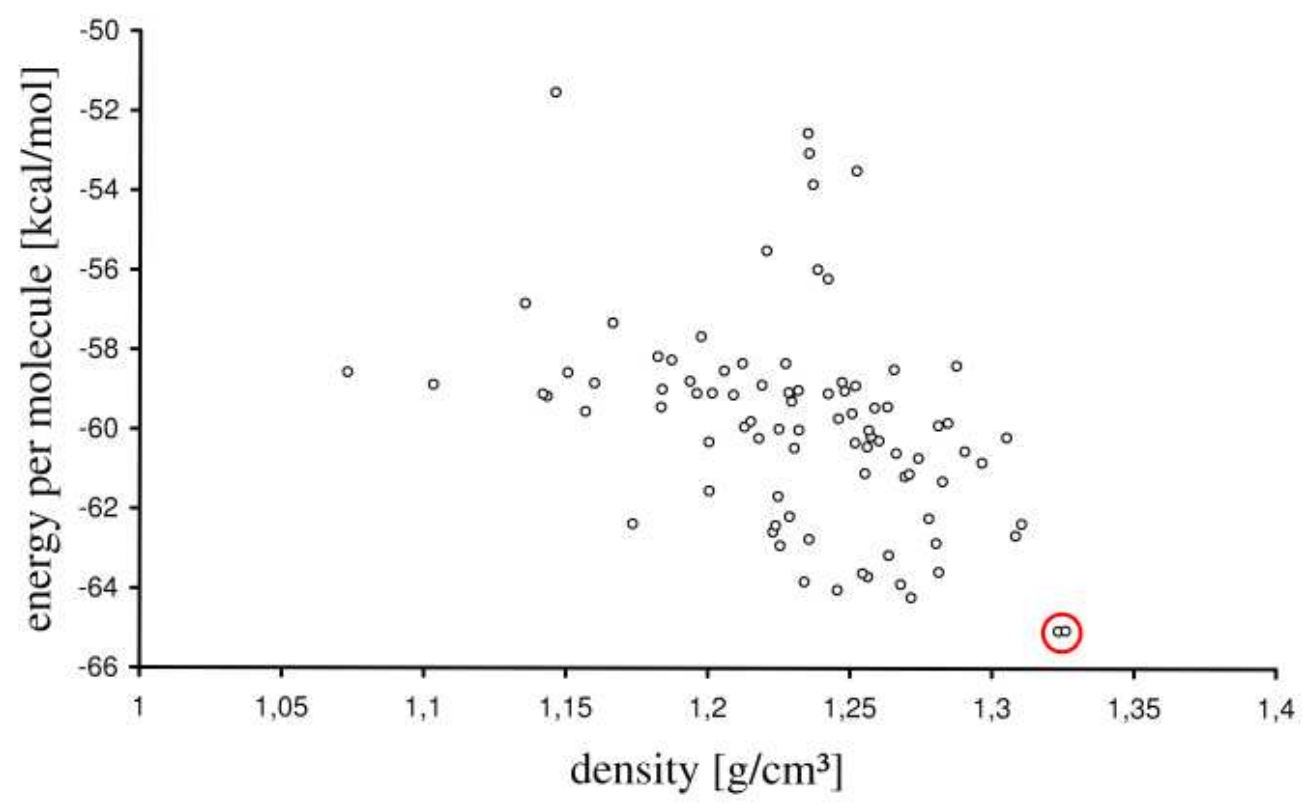

Figure 4. Energy-density plot in the space group P21/c (Cerius ${ }^{2}$ runs). Packings corresponding to phase I and phase III are circled. $28 \times 17 \mathrm{~mm}(600 \times 600 \mathrm{DPI})$ 
a)

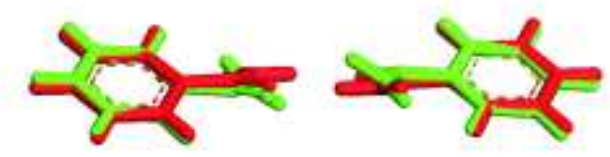

b)

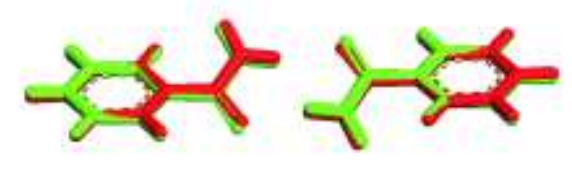

Figure 5. Overlay of the Cerius ${ }^{2}$ predicted (red) and observed (green) dimers of a) phase I and b) phase III.

$21 \times 7 \mathrm{~mm}(600 \times 600 \mathrm{DPI})$ 
Figure 6. Experimental powder diffraction pattern (blue) combined with the calculated diffraction data of the predicted phases I (red) and III (green). $30 \times 19 \mathrm{~mm}(600 \times 600 \mathrm{DPI})$ 


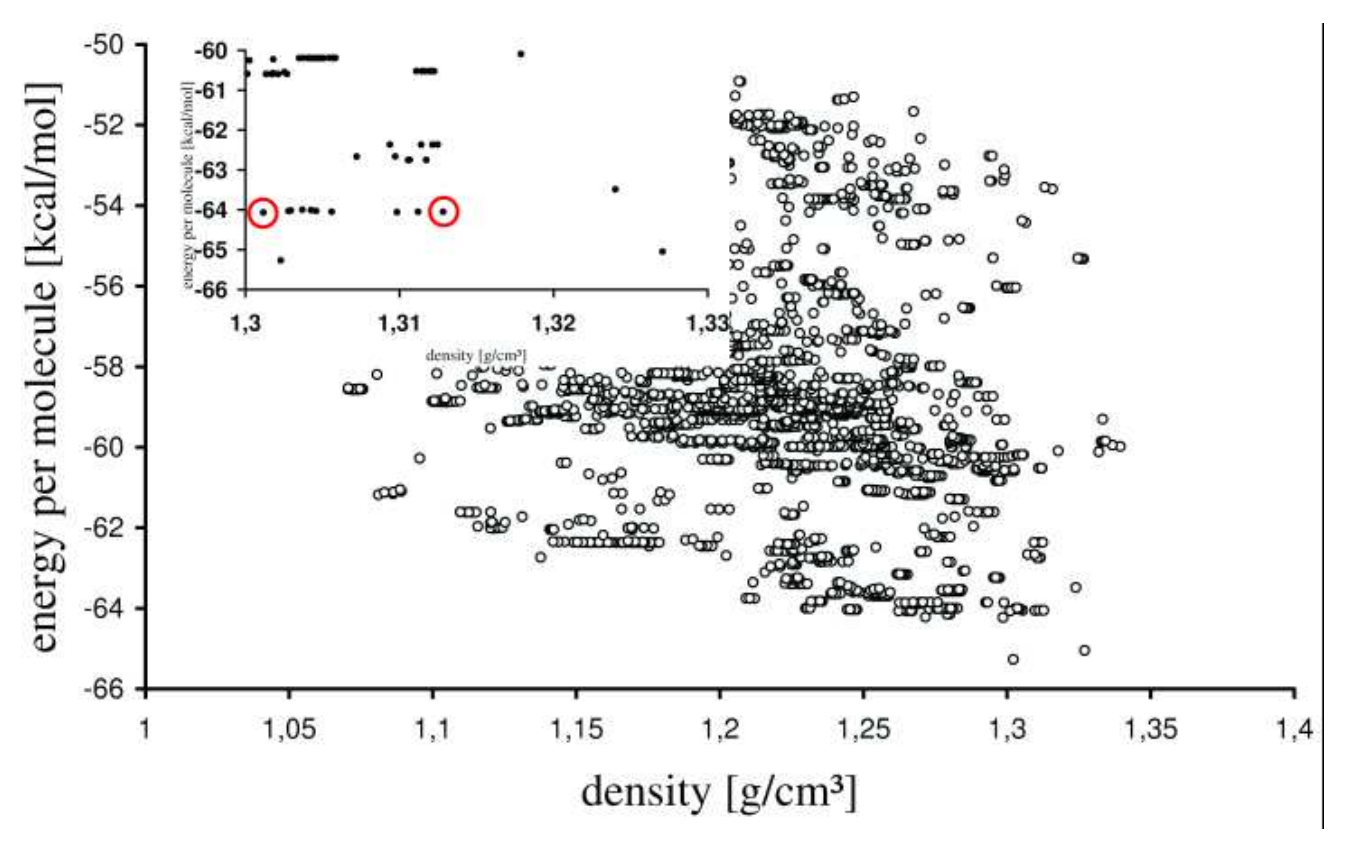

Figure 7. Energy-density plot in the space group P21/c. (MS Modeling runs). The inset shows a close up of the high density and low energy region. Packings corresponding to phase I and phase III are circled.

$30 \times 18 \mathrm{~mm}(600 \times 600 \mathrm{DPI})$ 
Page 29 of 33

$$
\begin{aligned}
& 1 \\
& 2 \\
& 3 \\
& 4 \\
& 5 \\
& 6 \\
& 7 \\
& 8 \\
& 9 \\
& 10 \\
& 11 \\
& 12 \\
& 13 \\
& 14 \\
& 15 \\
& 16 \\
& 17 \\
& 18 \\
& 19 \\
& 20 \\
& 21 \\
& 22 \\
& 23 \\
& 24 \\
& 25 \\
& 26 \\
& 27 \\
& 28 \\
& 29 \\
& 30 \\
& 31 \\
& 32 \\
& 33 \\
& 34 \\
& 35 \\
& 36 \\
& 37 \\
& 38 \\
& 50 \\
& 39 \\
& 40 \\
& 53 \\
& 53 \\
& 4 \\
& 42 \\
& 43 \\
& 43 \\
& 45 \\
& 45 \\
& 46 \\
& 47 \\
& 48 \\
& \hline
\end{aligned}
$$

a)

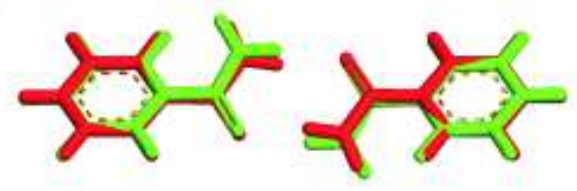

b)

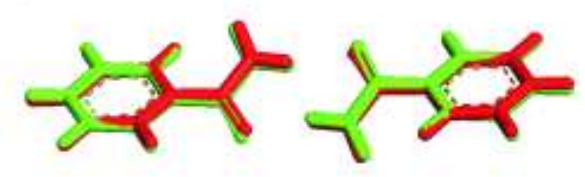

Figure 8. Overlay of the MS Modeling predicted (red) and observed (green) dimers of a) phase I and

b) phase III.

$23 \times 7 \mathrm{~mm}(600 \times 600 \mathrm{DPI})$

http://mc.manuscriptcentral.com/tandf/jenmol 
a)

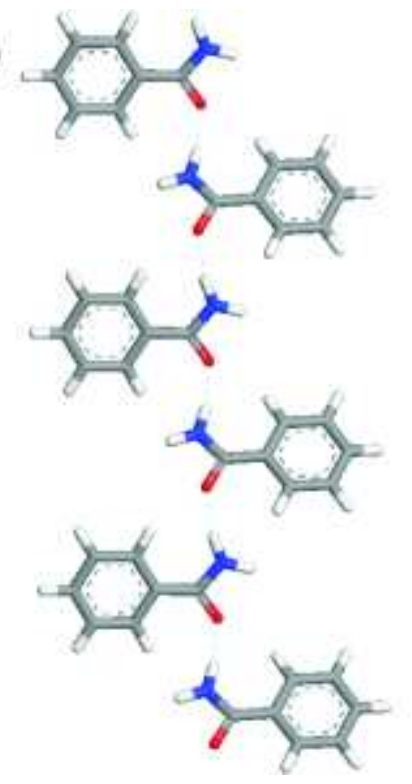

b)

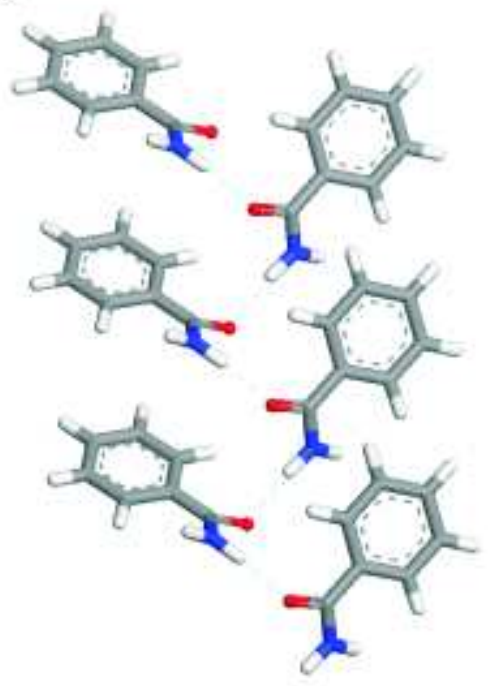

Figure 9. Hydrogen bonding pattern in the predicted structures with a) the lowest energy and b) the highest density. $19 \times 12 \mathrm{~mm}(600 \times 600 \mathrm{DPI})$ 


\section{Page 31 of 33}

1

2

3

4

5

6

7

8

9

10

11

12

13

14

15

16

17

18

19

20

21

22

23

24

25

26

27

28

29

30

31

32

33

34

35

36

37

38

39

40

41

42

43

44

45

46

47

48

49

50

51

52

53

54

55

56

57

58

59

60

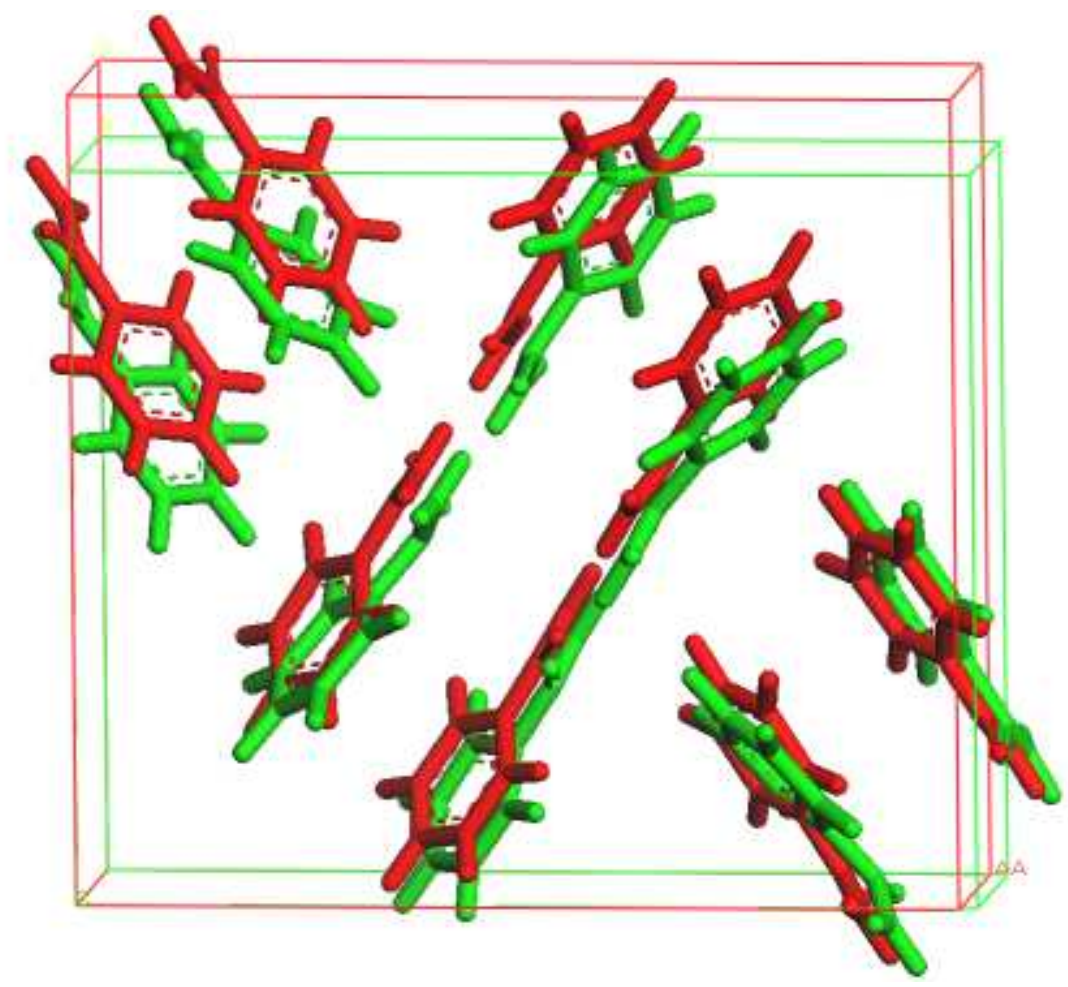

Figure 10. Overlay of the optimised (red) and experimental (green) unit cell of phase II. $167 \times 131 \mathrm{~mm}(72 \times 72 \mathrm{DPI})$ 

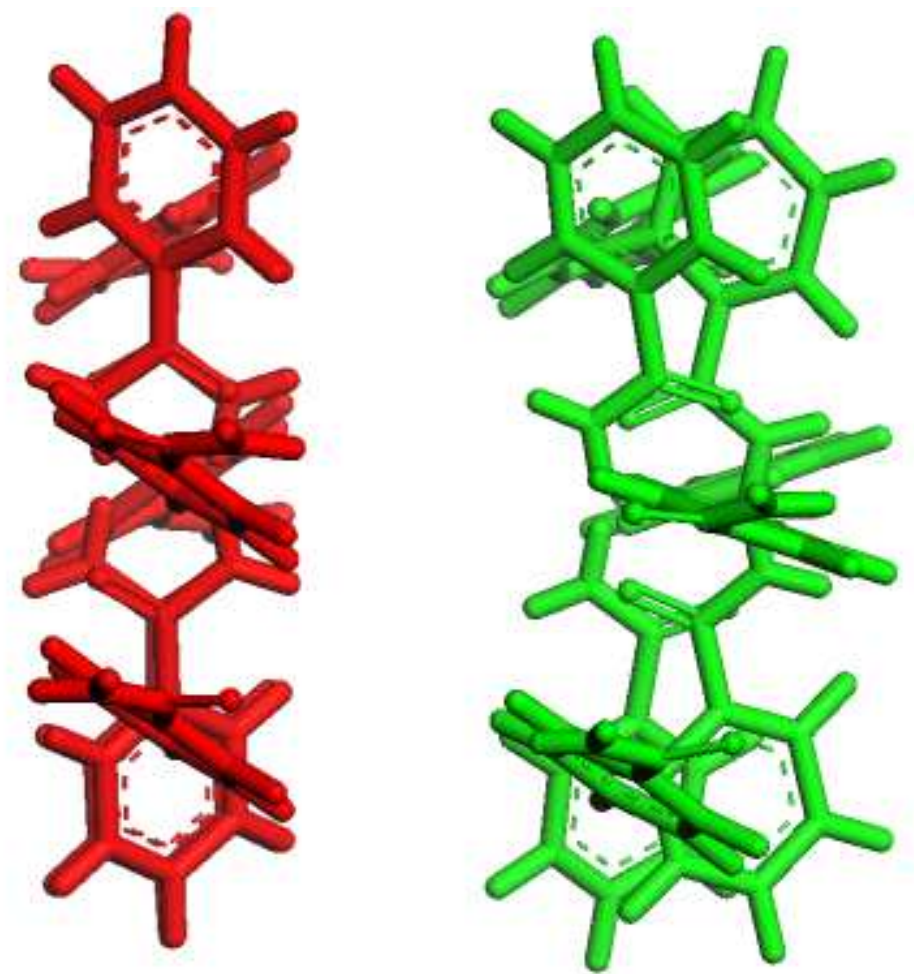

Figure 11. View perpendicular onto a pair of dimers of benzamide as found in the crystal structure of phase II before (green) and after (red) energy minimisation. $167 \times 131 \mathrm{~mm}(72 \times 72 \mathrm{DPI})$ 
1

2

3

4

5

6

7

8

9

10

11

12

13

14

15

16

17

18

19

20

21

22

23

24

25

26

27

28

29

30

31

32

33

34

35

36

37

38

39

40

41

42

43

44

45

46

47

48

49

50

51

52

53

54

55

56

57

58

59

60

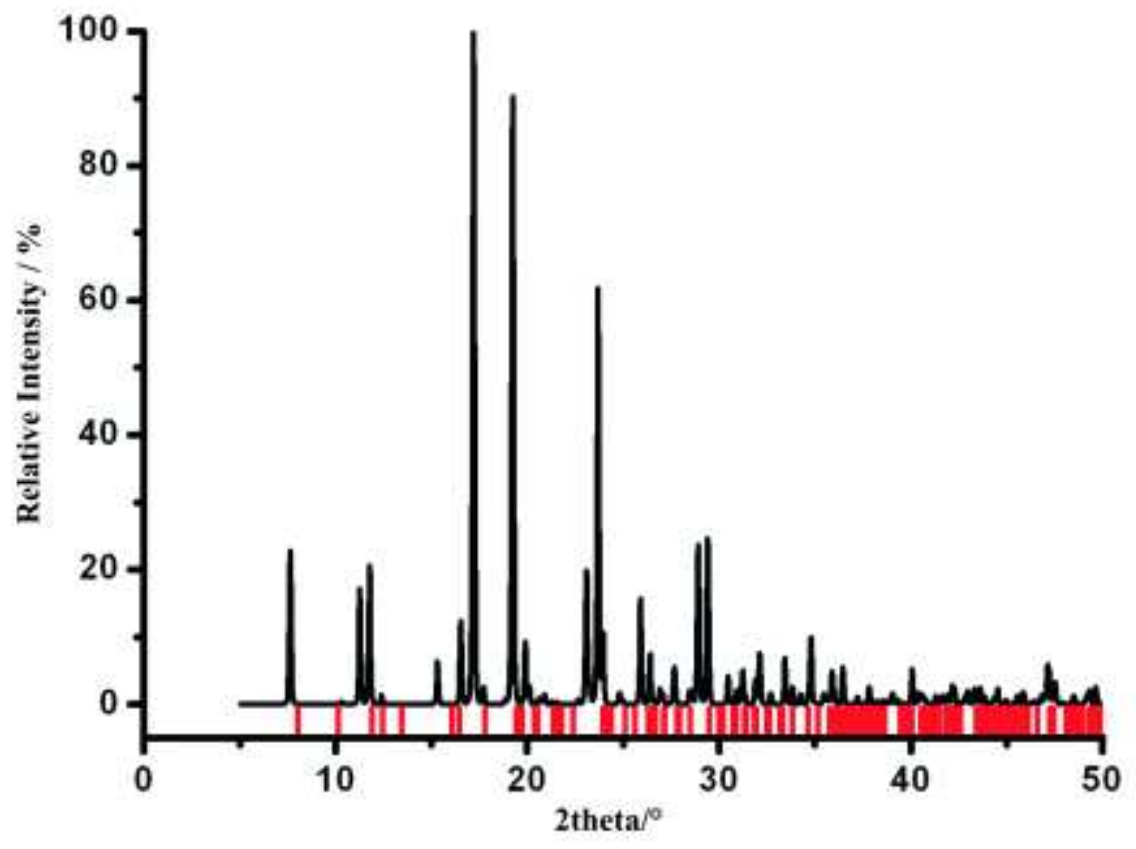

Figure 12. Simulated powder pattern of the minimised structure and ticks (red) of the experimentally known phase II. $40 \times 30 \mathrm{~mm}$ (300 x 300 DPI) 